\title{
Vascular Flora of a Red Sandstone Hills Site Canadian County, Oklahoma
}

\author{
Bruce W. Hoagland \\ Oklahoma Biological Survey \\ and Department of Geography \\ University of Oklahoma \\ Norman, OK 73019 \\ e-mail: bhoagland@ou.edu
}

\author{
Amy Buthod \\ Oklahoma Biological Survey \\ and Department of Botany and Microbiology \\ University of Oklahoma \\ Norman, OK 73019
}

This article reports the results of an inventory of the vascular plants from a site in central Oklahoma. Three hundred thirty-four species of vascular plants in 237 genera and 76 families were collected. The most species were collected from the families Poaceae (56) and Asteraceae (54). The genera with the most species were Euphorbia and Eragrostis, both with six species. One hundred six species were annuals, 227 perennials, and 1 biennial. Forty-nine species of woody plants were present. Forty-one species, or $12.3 \%$ of the flora, were exotic to Oklahoma. No species listed as threatened or endangered by the U.S. Fish and Wildlife Service were encountered, but two species (Escobaria vivipara and Mublenbergia bushii) are tracked by the Oklahoma Natural Heritage Inventory.

\section{INTRODUCTION}

Canadian County has been the focus of botanical research for several decades. The first plant collections from the county were made by Shultz and Sawyer on January 10, 1917, when they collected Helianthus maximiliani, Oenothera rhombipetala, Salsola iberica, and Senecio riddellii. There was no further collecting until 1926, but in the 1930s botanical studies began in ernst. Botanical collections in Canadian County have focused on the "Caddo Canyons" in the western third of the county. Of the 1,121 species recorded in the Oklahoma Vascular Plants database (Hoagland et al. 2006) for Canadian County, 964 are from canyons such as Devil's / Methodist, Grocket, Waters, and Widowmaker. On 29 May 1933, J. C. Shirley collected 38 specimens from Devil's Canyon. Elbert Little collected 87 species from Devil's Canyon in September, 1936. Several collectors visited Devils Canyon in 1937, but the most prolific was Milton Hopkins, who collected 145 in April and September of 1937 (Hoagland et al. 2006). Although collecting continued in Devil's Canyon, emphasis shifted to nearby Water Canyon when Connie Taylor gathered 321 specimens (see Taylor [1961] for a complete species list). Botanical collections outside the canyons have not focused on a particular locale in the county. Although 1,121 taxa of vascular plants have been reported from Canadian County, it is fewer than the taxa reported from adjacent Oklahoma (1,399) and Cleveland $(1,426)$ counties. The current project was initiated on the assumption that focused collection effort at a given site would yield additional county records. It will also provide land managers with a working species list to help guide their activities.

\section{STUDY AREA}

The study area encompasses 64.7 ha in Canadian County (Figure) along the Canadian River. Latitudinal extent ranges from $35.34^{\circ} \mathrm{N}$ to $35.36^{\circ} \mathrm{N}$ and longitudinal extent from $97.67^{\circ} \mathrm{W}$ to $97.68^{\circ} \mathrm{W}$. The study area is located within the subtropical humid (Cf) climate zone (Trewartha 1968). Summers are 
warm (mean July temperature $=27.8^{\circ} \mathrm{C}$ ) and humid, whereas winters are relatively short and mild (mean January temperature $=$ $\left.1.78^{\circ} \mathrm{C}\right)$. Mean annual precipitation is 87.6 $\mathrm{cm}$, with periodic severe droughts (Oklahoma Climatological Survey 2006).

Physiographically, the study area is located within the Osage Plains section of the Central Lowlands province (Hunt 1974) and the Central Redbed Plains province of Oklahoma (Curtis and Ham 1979). The surface geology is primarily Permian red sandstone and shale with Quaternary silt, sand, and clay along the Canadian River floodplain (Branson and Johnson 1979). Elevation ranges from 411.5 $\mathrm{m}$ to $358.7 \mathrm{~m}$. The predominant potential vegetation types are Tallgrass Prairie and Bottomland vegetation (Duck and Fletcher 1943).

\section{METHODS}

Collections were made monthly from March to June during the 2005 and 2006 growing seasons. The predominant vegetation association at the site were ascribed according to Hoagland (2000) and attributed to each collection. Vouchers for species exotic to North America were made from naturalized populations only, thus excluding cultivated and ornamental plants. Specimens were processed at the Robert Bebb Herbarium of the University of Oklahoma (OKL) following standard procedures. Manuals used for specimen identification included Waterfall (1969), Barkley (1986), and Diggs et al. (1999). Origin, either native or introduced, was determined by using Taylor and Taylor (1991) and US Department of Agriculture-Natural Resources Conservation Service (USDA-NRCS 2006). Nomenclature follows the USDA-NRCS (2006). Voucher specimens were deposited at OKL.

\section{RESULTS AND DISCUSSION}

Three hundred thirty-four species of vascular plants in 237 genera and 76 families were collected (appendix 1). The greatest numbers of species were from the Asteraceae (56) and Poaceae (54). The largest genera were Euphorbia and Eragrostis each with 6 species. There were three species of ferns, two gymnosperm, 78 monocots, and 252 dicots (Table). One hundred and six species were annuals, 227 perennials, and one biennial. Forty-nine species of woody plants were present. The absence of $Q$. stellata at the site is noteworthy. This study contributed an additional 84 species to the flora of Canadian County for a total of 1,205 species.

Forty-one species or $12.3 \%$ of the flora was non-native to North America. The Poaceae and Fabaceae had the greatest number of exotic species, 12 and 7 respectively. There were three exotic species in the genus Bromus and two in Ulmus. These values are consistent with other floristic studies from Oklahoma, in which exotic species constitute $9 \%-15 \%$ of the flora (Hoagland and Buthod 2003, Hoagland and Buthod 2004, Hoagland and Johnson 2001, Hoagland and Johnson 2004a, Hoagland and Johnson 2004b, Hoagland et al. 2004a, Hoagland et al. 2004b, Hoagland and Wallick 2003,). An exception is Red Slough and Grassy Slough, where exotic species constituted 6.6\% (Hoagland and Johnson, 2004b).

No species listed as threatened or endangered by the U.S. Fish and Wildlife Service were encountered. However, Escobaria vivipara (G5S2S3) and Mublenbergia bushii (G5S1S2), which are tracked by the Oklahoma Natural Heritage Inventory (2006), were present. Species are ranked by the ONHI according to level of imperilment at the global $[\mathrm{G}]$ and state $[\mathrm{S}]$ level on a scale of 
pecies that is imperiled and 5 a species that is secure

[Groves et al. 1995]).

Four vegetation associations/land cover types occurred at the study area. Each is described below with a brief list of associated species.

\section{Schizachyrium scoparium - Boutelona curtipendula} herbaceous association occurred on shallow sandstone derived soils and was the predominant grassland vegetation at the site. Associated species included Argythamnia mercurialiana, Baptisia australis, Boutelona birsuta, Dalea aurea, D. enneandra, D. multiflora, $D$. purpurea, Euphorbia corollata, Hedeoma drummondii, Ipomoea leptophylla, Krameria lanceolata, Lesquerella ovalifolia, Linum rigidum, Paronychia jamesii, Penstemon cobaea, Polygala alba, Stillingia sylvatica, and Tradescantia occidentalis.

\section{Quercus mueblenbergii - Juniperus virginiana} forest association is a vegetation type not described in Hoagland (2000), but was the predominant forest vegetation at Camp Kickapoo. Two subtypes most likely exist, although further analysis is necessary. The first occupies drier habitats and is characterized by associated species such as Carex albicans, Cornus drummondii, Opuntia macrorbiza, Q. marilandica, Rhus aromatica, Ruellia bumilis, Sideroxylon lanuginosum, Symphoricarpos orbiculatus, Tridens flavus, and Viburnum rufidulum. The moist or mesic subtypes occurred along streams and deep gullies. Associated species include Arisaema dracontium, Bromus pubescens, Chasmanthium latifolium, Desmodium glutinosum, Elephantopus tomentosus, Juglans nigra, Phryma leptostachya, Polygonatum biflorum, Q. macrocarpa, Sanicula canadensis, Sapindus drummondii, and Verbesina alternifolia.
3. Wetland and aquatic vegetation was of restricted to a small human-made pond and swales on the Canadian River floodplain. Associated species included Amorpha fruticosa, Cephalanthus occidentalis, Echinochloa crus-galli, Polygonum lapathifolium, Symphyotrichum subulatum, and Typha domingensis

4. Disturbed areas and old-field vegetation included roadsides and areas exhibiting signs of physical disruption. Associated species included Capsella bursa-pastoris, Chamaesyce maculata, C. missurica, Erodium cicutarium, Geranium carolinianum, Lamium amplexicaule, Mollugo verticillata, Oenothera biennis, O. laciniata, Oxalis stricta, Phytolacca americana, Portulaca pilosa, Sherardia arvensis, Solanum dimidiatum, $S$. elaeagnifolium, Sorghum balepense and Viola bicolor.

\section{REFERENCES}

Barkley, T. M., (ed.) 1986. Flora of the Great Plains. Lawrence (KS): University Press of Kansas. 1,392 p.

Branson, C. C. and K. S. Johnson. 1979. Generalized geologic map of Oklahoma. In: Johnson K. S., C. C. Branson, N. M. Curtis, W. E. Ham, W. E. Harrison, M. V. Marcher, and J. F. Roberts, editors. Geology and earth resources of Oklahoma. Oklahoma Geological Survey, Norman. p 4.

Curtis, N. M. and W. E. Ham. 1979. Geomorphic provinces of Oklahoma. In: Johnson K. S., C. C. Branson, N. M. Curtis, W. E. Ham, W. E. Harrison, M. V. Marcher, and J. F. Roberts, editors. Geology and earth resources of Oklahoma. Oklahoma Geological Survey, Norman. p 5.

Diggs G. M., B. L. Lipscomb, and R. J. O'Kennon. 1999. Shinners and Mahler's illustrated flora of North 
Central Texas. Botanical Research

Institute of Texas and Austin College, Fort Worth. $1626 \mathrm{p}$.

Duck, L. G. and J. B. Fletcher. 1943. A game type map of Oklahoma. A survey of the game and furbearing animals of Oklahoma. Oklahoma Department of Wildlife Conservation, Oklahoma City.

Groves C. R., M. L. Klein, and T. F. Breden. 1995. Natural Heritage Programs: public-private partnerships for biodiversity conservation. Wildlife Society Bulletin 23:784-790.

Hoagland, B. W. 2000. The vegetation of Oklahoma: a classification of landscape mapping and conservation planning. Southwest Naturalist 45:385-420.

Hoagland, B. W. and A. K. Buthod. 2003. Vascular flora of the Keystone Wildlife Management Area, Creek, Pawnee, and Osage Counties, Oklahoma. Oklahoma Native Plant Record 3:23-37.

Hoagland, B. W. and A. K. Buthod. 2004. Vascular flora of Hugo Lake Wildlife Management Area, Choctaw County, Oklahoma. Southeastern Naturalist 30: 701-714.

Hoagland, B. W. and F. L. Johnson. 2001. Vascular flora of the Chickasaw National Recreation Area, Murray County, Oklahoma. Castanea 66:383400.

Hoagland B. W. and F. L. Johnson. 2004a. Vascular Flora of Chouteau Wildlife Management Area, Wagoner County, Oklahoma. Oklahoma Native Plant Record 4:30-39.

Hoagland, B. W. and F. L. Johnson. 2004b. Vascular flora of Red Slough and Grassy Slough Wildlife Management Areas, Gulf Coastal Plain, McCurtain
County, Oklahoma. Castanea 69:284296.

Hoagland, B. W and K. Wallick. 2003. Vascular flora of Oologah Wildlife Management Area, Nowata County, Oklahoma. Proceedings of the Oklahoma Academy of Science 83:4762.

Hoagland, B. W., A. K. Buthod and W. Elisens. 2004a. Vascular flora of Washita Battlefield National Historic Site, Roger Mills County, Oklahoma. Sida 21:1187-1197.

Hoagland, B. W., P. Crawford-Callahan, P. Crawford, and F. L. Johnson. 2004b. Vascular flora of Hackberry Flat, Fredrick Lake, and Suttle Creek, Tillman County, Oklahoma. Sida 21 429-445.

Hoagland, B., A. K. Buthod, I. Butler, P. Callahan-Crawford, W. Elisens, A. Udasi, and R. Tyrl. 2006. Oklahoma Vascular Plants Database. [online]. Available: http://www.biosurvey.ou.edu. (Accessed on 1 March 2005).

Hunt, C. B. 1974. Natural Regions of the United States and Canada. W. H. Freeman, San Francisco. 725 p. Oklahoma Climatological Survey. 2005. Oklahoma Climatological Data [online]. (accessed on 1 March 2005). Available from http://www.ocs.ou.edu/.

Oklahoma Natural Heritage Inventory. 2006. ONHI working list of rare Oklahoma plants [online]. (accessed on 1 March 2005). Available from http://www.biosurvey.ou.edu/publica t.html.

Palmer, M. W., G. L. Wade, and P. Neal. 1995. Standards for the writing of floras. Bioscience 45:339-345.

\section{Hoagland \& Buthod}


USDA-NRCS. 2005. The PLANTS database [online]. (Accessed on 1 March 2005). Available from http://plants.usda.gov/plants. National Plant Data Center, Baton Rouge, LA.

Waterfall, U. T. 1969. Keys to the flora of Oklahoma, $4^{\text {th }}$ ed. Published by the author, Stillwater. $246 \mathrm{p}$.

Trewartha, G. T. 1968. An introduction to climate. McGraw-Hill, New York. 399 p.

Table Summary of floristic collections from a study site in Canadian County, Oklahoma.*

\begin{tabular}{|c|c|c|c|}
\hline Taxonomic group & Species & Native spp. & Introduced spp. \\
\hline Pteridophyta & 3 & 3 & 0 \\
\hline Coniferophyta & 2 & 1 & 1 \\
\hline Magnoliophyta & 329 & 289 & 40 \\
\hline Magnoliopsida & 250 & 222 & 28 \\
\hline Liliopsida & 79 & 67 & 12 \\
\hline Total & 334 & 293 & 41 \\
\hline
\end{tabular}

* Table format follows Palmer et al. (1995). 


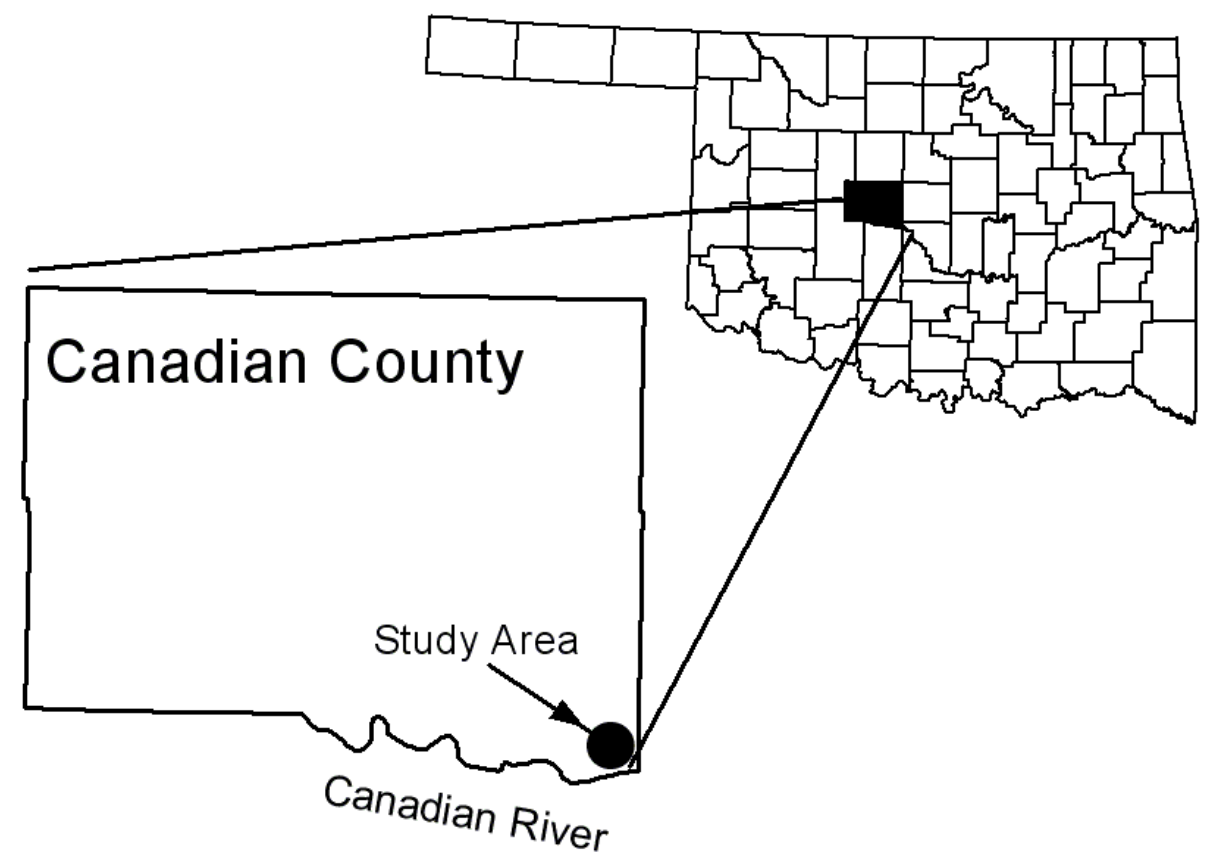

Figure Location of the Canadian County study area. 


\section{APPENDIX}

Annotated species list. The first entry is habitat (QMJV-M = Quercus mueblenbergii - Juniperus virginiana forest association, mesic subtype; QMJV-X $=$ Q. mueblenbergii - J. virginiana forest association, xeric subtype; SSBC = Schizachyrium scoparium - Boutelona curtipendula herbaceous association, WETL = wetland and aquatic vegetation, $\mathrm{DAOF}=$ disturbed areas and old-field vegetation), followed by life history $(\mathrm{A}=$ annual, $\mathrm{B}=$ biennial, $\mathrm{P}=$ perennial $)$, and collection number. Exotic species are denoted with an asterisk. Voucher specimens were deposited at the Robert Bebb Herbarium at the University of Oklahoma (OKL).

\section{PTERIDOPHYTA}

\section{Dryopteridaceae}

Woodsia obtusa (Spreng.) Torr.(bluntlobe cliff fern) - QMSJV-M; P; H; AB-6788

\section{Ophioglossaceae}

Botrychium virginianum (L.) Sw. (Rattlesnake fern) - QMJV-M; P; H; AB-6766

\section{Pteridaceae}

Pellaea atropurpurea (L.) Link (purple cliffbreak) - QMJV-X; P; H; AB-6261

\section{CONIFEROPHYTA}

\section{Cupressaceae}

Juniperus virginiana L. (Eastern redcedar) QMJV-X; P; T; AB-6206

Thuja occidentalis L.* (arborvitae) - DAOF; P; T; AB-6630

\section{MAGNOLIOPHYTA Magnoliopsdia}

\section{Acanthaceae}

Ruellia bumilis Nutt. (wild petunia)- QMJV-X, SSBC; P; H; Ab-6559

\section{Aceraceae}

Acer negundo L. (boxelder) - QMJV-M,

WETL; P; T; AB-6209

\section{Amaranthaceae}

Amaranthus palmeri S. Wats. (Carlessweed) DAOF; A; H; AB-6608

Iresine rbizomatosa Standl. (Juda's bush) - QSJVM; P; H; AB-6584

\section{Anacardiaceae}

Rhus aromatica Ait. (fragrant sumac)- QMJVX; P; S; AB-6887

R. glabra L. (smooth sumac) - DAOF, QMJVX; P; S; AB-6204

Toxicodendron radicans (L.) Kuntze (poison ivy) - QMJV-M; P; S; AB-6252

\section{Apiaceae}

Chaerophyllum tainturieri Hook. (hairyfruit chervil) - DAOF; A; H; AB-6922

Sanicula canadensis L. (blacksnakeroot) QMJV-M; P; H; AB-6198

Spermolepis echinata (Nutt. ex DC.) Heller

(bristly scaleseed) - SSBC; A; H; AB-6861

Torilis arvensis (Huds.) Link* (spreading hedgeparsley) - DAOF; A; H; AB-7148

\section{Apocynaceae}

Apocynum cannabinum L. (Indianhemp) - SSBC; P; H; AB-6791

\section{Asclepiadaceae}

Asclepias amplexicaulis $\mathrm{Sm}$. (clasping milkweed) - WETL; P; H; AB-6806

A. stenophylla Gray (slimleaf milkweed) SSBC; P; H; AB-6555

A. viridiflora $\mathrm{Raf}$. (green comet milkweed) SSBC; P; H; AB-7149

A. viridis Walt. (green antelopehorn) - DAOF, SSBC; P; H; AB-6821

\section{Asteraceae}

Achillea millefolium L. (common yarrow) -

DAOF, SSBC; P; H; AB-6775 
Ambrosia psilostachya DC. (Plains ragweed) -

DAOF, SSBC; P; H; AB-6551

A. trifida L. (giant ragweed) - DAOF; A; $\mathrm{H}$; AB-6233

Amphiachyris dracunculoides (DC.) Nutt. (prairie broomweed) - DAOF; A; H; AB-6254

Aphanostephus skirrhobasis (DC.) Trel. (Arkansas dozedaisy) - SSBC; A; H; AB6800

Artemisia ludoviciana Nutt. (white sagebrush) SSBC; P; H; AB-6631

Baccharis salicina Torr. \& Gray (false willow) WETL; P; S; AB-6548

Bidens bipinniata L. (Spanish needles) - DAOF, QMJV-m; A; H; AB-6585

Carduus nutans L.* (nodding thistle) - DAOF; B; H; AB-6801

Chrysopsis pilosa Nutt. (goldenaster) - SSBC; A; $\mathrm{H}$; AB-6889

Cirsium altissimum (L.) Hill (tall thistle) QMJV-M; P; H; AB-6599

C. undulatum (Nutt.) Spreng. (wavyleaf thistle) - SSBC; P; H; AB-7166

Conyza canadensis (L.) Cronq. (Canadian horseweed) - DAOF; A; H; AB-6894

C. ramosissima Cronq. (dwarf horseweed) DAOF; A; H; AB-6244

Echinacea angustifolia DC. (Blacksamson) SSBC; P; H; AB-6811

Elephantopus carolinianus Raeusch. (Carolina elephantsfoot) - QMJV-M; P; H; AB6629

Engelmannia peristenia (Raf.) Goodman \& Lawson (Engelmann's daisy) - SSBC; P; $\mathrm{H}$; AB-6787

Erigeron strigosus Muhl. ex Willd. (prairie fleabane) - QMJV-M; A; H; AB-6552

Eupatorium serotinum Michx. (thoughtwort)QMJV-M, WETL; P; H; AB-6632

Evax prolifera Nutt. ex DC. (bighead pygmycudweed) - DAOF; A; H; AB-6860

E. verna Raf. (spring pygmycudweed) DAOF; A; H; AB-6938
Gaillardia aestivalis (Walt.) H. Rock (lanceleaf blanketflower) - SSBC; P; H; AB-6633

G. suavis (Gray \& Engelm.) Britt. \& Rusby (perfumeballs) - DAOF, SSBC; P; H; AB6218

Gamochaeta purpurea (L.) Cabrera (purple everlasting) - DAOF: P; H; AB-6769

Grindelia papposa Nesom \& Sun (Spanish gold) - SSBC; A; H; AB-6553

Gutierrezia sarothrae (Pursh) Britt. \& Rusby (broom snakeweed) - DAOF, SSBC; P; H; AB-6588

Helianthus annuus L. (common sunflower) DAOF; A; H; AB-6219

H. petiolaris Nutt. (prairie sunflower) - SSBC; A; H; AB-6590

Heterotheca subaxillaris (Lam.) Britt. \& Rusby (camphorweed) - SSBC; A; H; AB-6616

Hieracium longipilum Torr. (hairy hawkweed) QMJV-X; P; H; AB-6872

Hymenopappus tenuifolius Pursh (Chalk Hill hymenopappus) - SSBC; P; H; AB-6592

Iva annua $\mathrm{L}$. (annual marshelder) - WETL; A; $\mathrm{H}$; AB-6202

Lactuca canadensis L. (Canada lettuce) - DAOF; A; H; AB-6900

Liatris mucronata DC. (cusp blazing star) SSBC; P; H; AB-6596

L. squarrosa (L.) Michx. (scaly blazing star) SSBC; P; H; AB-6595

Machaeranthera pinnatifida (Hook.) Shinners (Lacy tansyaster) - SSBC; P; H; AB-6278

Oligoneuron rigidum (L.) Small var. rigidum (stiff goldenrod) - QMJV-M; P; H; AB-6598

Palafoxia rosea (Bush) Cory (rosy palafox) SSBC; A; H; AB-6549

Pyrrhopappus grandiflorus (Nutt.) Nutt. (tuberous desert-chicory) - DAOF; P H; AB-6919

Ratibida columnifera (Nutt.) Woot. \& Standl. (upright prairie coneflower) - SSBC; P; H; AB-6269

Rudbeckia hirta L. (blackeyed susan) - SSBC; P; $\mathrm{H}$; AB-6257 
Silphium laciniatum L. (compassplant) - SSBC; $\mathrm{P} ; \mathrm{H}$; AB-6223

Solidago missouriensis Nutt. (Missouri goldenrod) - SSBC; P; H; AB-6550

Sonchus asper (L.) Hill* (spiny sowthistle) DAOF; A; H; AB-6852

Symphyotrichum drummondii (Lindl.) Nesom var. drummondii (Drummond's aster) - QMJVM; P; H; AB-6597

S. subulatum (Michx.) Nesom (annual saltmarsh aster) - WETL; A; H; AB-6273

Taraxacum officinale G. H. Weber ex Wiggers* (common dandelion) - DAOF; P; H; AB7155

Thelesperma filifolium (Hook.) Gray var. filifolium (stiff greenthread) - SSBC; P; H; AB-6617

Tragopogon dubius Scop.* (yellow salsify) DAOF; A; H; AB-6907

$V$ erbesina alternifolia (L.) Britt. ex Kearney (wingstem) - QMJV-M; P; H; AB-6601

$V$. encelioides (Cav.) Benth. \& Hook. f. ex Gray (golden crownbeard) - DAOF, SSBC; P; H; AB-6222

V. virginica L. (white crownbeard) - QMJV-M; P; H; AB-6255

Vernonia baldwinii Torr. (Baldwin's ironweed) SSBC; P; H; AB-6600

Xanthium strumarium L. (rough cocklebur) DAOF, WETL; A; H; AB-6225

\section{Bignoniaceae}

Campsis radicans (L.) Seem. ex Bureau (trumpet creeper) - DAOF; P; V; AB-7152

Catalpa bignonioides Walt. (southern catalpa) DAOF; P; T; AB-6790

C. speciosa (Warder) Warder ex Engelm. (northern catalpa) - DAOF; P; T; AB6611

\section{Boraginaceae}

Hackelia virginiana (L.) I. M. Johnston (beggarslice) - QMJV-M; P; H; AB-6201
Lithospermum caroliniense (Walt. Ex J. F. Gmel.) MacM. (Carolina puccoon) - SSBC; P; H; AB-6916

L. incisum Lehm. (narrowleaf stoneseed) DAOF, SSBC; P; H; AB-7168

Myosotis macrosperma Engelm. (largeseed forgetme-not) - QMJV-M; A; H; AB-6842

\section{Brassicaceae}

Brassica rapa L.* (field mustard) - DAOF; A; $\mathrm{H}$; AB-6816a

Camelina microcarpa DC.* (littlepod false flax) DAOF; A; H; AB-6823

Capsella bursa-pastoris (L.) Medik.* (shepherd's purse) - DAOF; A; H; AB-6924

Descurainia pinnata (Walt.) Britt. (western tansymustard) - DAOF; A; H; AB-6942

Draba brachycarpa Nutt. ex Torr. \& Gray (shortpod draba) - DAOF; A; H; AB-6943

Lepidium virginicum L. (Virginia pepperweed) DAOF; A; H; AB-6935

Lesquerella ovalifolia Rydb. ex Britt. subsp. alba (Goodman) Rollins \& Shaw (roundleaf bladderpod) - SSBC; P; H; AB-6840

\section{Cactaceae}

Escobaria missouriense (Sweet) D. R. Hunt (spinystar) - SSBC; P; H; AB-6960

Opuntia macrorbiza Engelm. (twistspine pricklypear) - QMJV-X, SSBC; P; S; AB6954

\section{Campanulaceae}

Triodanis biflora (Ruiz \& Pavón) Greene (clasping Venus' looking-glass) - DAOF, SSBC; A; H; AB-6940

\section{Caprifoliaceae}

Symphoricarpos orbiculatus Moench (coralberry) QMJV-X; P; S; AB-6236

Viburnum rufidulum Raf. (rusty blackhaw) QMJV-X; P; S; AB-6226

\section{Caryophyllaceae}

Arenaria serpyllifolia L.* (thymeleaf sandwort) DAOF; A; H; AB-6911 
Cerastium pumilum W. Curtis* (European chickweed) - DAOF; A; H; AB-6948

Paronychia jamesii Torr. \& Gray (James' nailwort) - SSBC; P; H; AB-6567

Stellaria media (L.) Vill.* (Common chickweed) - DAOF, QMJV-M;A; H; AB-6918

\section{Celastraceae}

Celastrus scandens L. (American bittersweet) QMJV-M; P; S; AB-6634

\section{Chenopodiaceae}

Chenopodium murale L.* (nettleleaf goosefoot) DAOF; A; H; AB-6565

C. pratericola Rydb. (desert goosefoot) DAOF; A; H; AB-6606

C. simplex (Torr. ) Raf. (mapleleaf goosefoot) DAOF; A; H; AB-6566

\section{Convolvulaceae}

Ipomoea leptophylla Torr. (bush morning-glory) SSBC; P; H; AB-6893

\section{Cornaceae}

Cornus drummondii C. A. Mey. (roughleaf dogwood) - DAOF, QMJV-X; P; T; AB6871

\section{Cucurbitaceae}

Cucurbita foetidissima Kunth (Missouri gourd) DAOF; P; H; AB-6883

Melothria pendula L. (Guadeloupe cucumber) DAOF; P; H; AB-6253

\section{Elaeagnaceae}

Elaeagnus angustifolia L.* (Russian olive) DAOF; P; T; AB-6558

\section{Euphorbiaceae}

Acalphya virginica L. (Virginia threeseed mercury) - DAOF; A; H; AB-6876

Argythamnia mercurialiana (Nutt.) Muell.-Arg. var. mercurilaiana (tall silverbush) - SSBC; $\mathrm{P} ; \mathrm{H} ; \mathrm{AB}-6815$

Chamaesyce glyptosperma (Englem.) Small (ribseed sandmat) - SSBC; A; H; AB-6618

C. maculata (L.) Small (spotted sandmat) DAOF; A; H; AB-6576
C. missurica (Raf.) Shinners (prairie sandmat) DAOF; A; H; AB-6897

C. nutans (Lag.) Small (eyebane) - DAOF; A; $\mathrm{H}$; AB-6247

Croton glandulosus L. (Vente conmigo) - SSBC; A; H; AB-6258

C. lindheimerianus Scheele (threeseed croton) SSBC; A; H; AB-6615

C. monanthogynus Michx. (prairie tea) - DAOF, SSBC; A; H; AB-6614

C. texensis (Klotzsch) Muell.-Arg. (Texas croton) - SSBC; A; H; AB-6593

Euphorbia corollata L. (flowering spurge) SSBC; P; H; AB-6260

E. cyathophora Murr. (fire-on-the-mountain) DAOF; A; H; AB-6604

E. dentata Michx. (toothed spurge) - DAOF; A; $\mathrm{H}$; AB-6241

E. hexagona Nutt. ex Spreng. (sixangle spurge)

- DAOF; A; H; AB-6249

E. marginata Pursh (snow-on-the-mountain) -

DAOF; A; H; AB-6216

E. spathulata Lam. (warty spurge) - DAOF; A; $\mathrm{H}$; AB-6844

Stillingia sylvatica Garden ex L. (Queen's delight) - SSBC; P; H; AB-6767

\section{Fabaceae}

Amorpha canescens Pursh (leadplant) - WETL; P; S; AB-6772

A. fruticosa L. (desert false indigo) - SSBC; P; S; AB-6272

Amphicarpaea bracteata (L.) Fern. (American hogpeanut) - QMJV-X; A; H; AB-7158

Astragalus lotiflorus Hook. (lotus milkvetch) QMJV-X; P; H; AB-6945

Baptisia australis (L.) R. Br. ex Ait. f. (blue wild indigo) - SSBC; P; H; AB-6773

Cercis canadensis L. (eastern redbud) - QMJVM, QMJV-X; P; T; AB-6279

Dalea aurea Nutt. ex Pursh (golden prairie clover) - SSBC; P; H; AB-6268

D. enneandra Nutt. (nineanther prairie clover) SSBC; P; H; AB-6229 
D. multiflora (Nutt.) Shinners (roundhead prairie clover) - SSBC; P; H; AB-6577

D. purpurea Vent. (purple prairie clover) SSBC; P; H; AB-6881

Desmanthus illinoensis (Michx.) MacM. ex B. L. Robins. \& Fern. (prairie bundleflower) DAOF, WETL; P; H; AB-6230

Desmodium canescens (L.) DC. (hoary ticktrefoil) - QMJV-M; P; H; AB-6568

D. glutinosum (Muhl.) ex Willd. Wood (pointedleaf ticktrefoil) - QMJV-M; P; H; AB-7164

D. nudiflorum (L.) DC. (nakedflower ticktrefoil) - QMJV-M; P; H; AB-6896

D. sessilifolium (Torr.) Torr. \& Gray (sessileleaf ticktrefoil) - SSBC; P; H; AB-6571

Gleditsia triacanthos L. (honeylocust) - DAOF; P; T; AB-7159

Gymnocladus dioicus (L.) K. Koch (kentucky coffeetree) - QMJV-M; P; T; AB-7159

Indigofera miniata Ortega (coastal indigo) SSBC; P; H; AB-6853

Kummerowia stipulacea (Maxim.) Makino* (Korean clover) - DAOF; A; H; AB-3232

Lespedera cuneata (Dum.-Cours.) G. Don* (Chinese lespedeza) - DAOF; P; H; AB6265

L. stuevei Nutt. (tall lespedeza) - QMJV-X; P; $\mathrm{H}$; AB-6562

Medicago minima (L.) L.* (burr medick) DAOF; A; H; AB-6846

Melilotus officinalis (L.) Lam.* (yellow sweetclover) - DAOF; A; H; AB-6792

Mimosa nuttallii (DC.) B. L. Turner (Nuttall's sensitive-briar) - SSBC; P; H; AB-6774

Neptunia lutea (Leavenw.) Benth. (yellow puff) - SSBC; P; H; AB-6959

Oxytropis lambertii Pursh (purple locoweed) SSBC; P; H; AB-6822

Pisum sativum L.* (garden pea) - DAOF; A; H; AB-6816
Psoralidium tenuiflorum (Pursh) Rydb.

(Slimflower scurfpea) - SSBC; P; H; AB6828

Robinia psendoacacia L.* (black locust) - DAOF, QMJV-X; P; T; AB-6899

Strophostyles helvula (L.) Ell. (trailing fuzzybean)

- DAOF; P; H; AB-6573

Vicia villosa Roth* (winter vetch) - DAOF; A; $\mathrm{H}$; AB-6817

\section{Fagaceae}

Quercus macrocarpa Michx. (bur oak) - QMJV-

M; P; T; AB-6275

Q. marilandica Muenchh. (blackjack oak) -

QMJV-X; P; T; AB-6771

Q. mueblenbergii Engelm. (chinkapin oak) -

QMJV-M, QMJV-X; P; T; AB-6207

\section{Geraniaceae}

Erodium cicutarium (L.) L'Hér. Ex Ait.* (redstem stork's beak) - DAOF; A; H;

AB-6913

Geranium carolinianum L. (Carolina geranium) DAOF; A; H; AB-6786

G. pusillum L.* (small geranium) - DAOF; A; $\mathrm{H}$; AB-6850

\section{Juglandaceae}

Juglans nigra L. (black walnut) - QMJV-M; P; T; AB-7147

\section{Krameriaceae}

Krameria lanceolata Torr. (trailing krameria) SSBC; P; H; AB-6803

\section{Lamiaceae}

Hedeoma drummondii Benth. (Drummond's false pennyroyal) - SSBC; P; H; AB-6610

H. hispida Pursh (rough false pennyroyal) SSBC; A; H; AB-6858

Lamium amplexicaule L.* (henbit) - DAOF; A; $\mathrm{H}$; AB-6926

Salvia azurea Michx. ex Lam. (azure blue sage) - SSBC; P; H; AB-6271

S. lyrata L. (lyreleaf sage) - QMJV-M; P; H; AB-6886 
Teucrium canadense L. (Canada germander) QMJV-M; P; H; AB-6215

\section{Linaceae}

Linum rigidum Pursh (stiffstem flax) - SSBC; A; $\mathrm{H}$; AB-6845

\section{Loasaceae}

Mentzelia oligosperma Nutt. ex Sims (chickenthief) - SSBC; P; H; AB-6221

\section{Malvaceae}

Callirhoe involucrata (Torr. \& Gray) Gray (purple poppymallow) - DAOF; P; H; AB-6799

\section{Menispermaceae}

Cocculus carolinus (L.) DC. (Carolina coralbead) - QMJV-M; P; H; AB-6199

Menispermum canadense L. (common moonseed) - QMJV-M; P; H; AB-6797

\section{Molluginaceae}

Mollugo verticillata L. (carpetweed) - DAOF; A; H; AB-6888

\section{Moraceae}

Maclura pomifera (Raf.) Schneid. (Osage orange) - DAOF, QMJV-X; P; T; AB-6195

Morus rubra L. (red mulberry) - QMJV-M; P; T; AB-6620

\section{Nyctaginaceae}

Mirabilis albida (Walt.) Heimerl (white four o'clock) - SSBC; P; H; AB-6578

M. nyctaginea (Michx.) MacM. (heartleaf four o'clock) - DAOF; P; H; AB-6833

\section{Oleaceae}

Fraxinus pennsylvanica Marsh. (green ash) QMJV-M; P; T; AB-6560

\section{Onagraceae}

Calylophus berlandieri Spach (Berlandier's sundrops) - SSBC; P; H; AB-6825

Gaura sinuata Nutt. ex Ser. (wavyleaf beeblossom) - SSBC; P; H; AB-6827

Oenothera biennis L. (common eveningprimrose) - DAOF; P; H; AB-6213
O. laciniata Hill (cutleaf evening-primrose) DAOF; P; H; AB-6785

O. macrocarpa Nutt. (bigfruit evening-primrose) - SSBC; P; H; AB-6781

O. speciosa Nutt. (pinkladies) - SSBC; P; H; AB-6851

Stenosiphon linifolius (Nutt. ex James) Heynh. (false gaura) - SSBC; P; H; AB-6234

\section{Oxalidaceae}

Oxalis stricta $\mathrm{L}$. (common yellow oxalis) -

DAOF, SSBC; P; H; AB-6923

\section{Papaveraceae}

Argemone polyanthemos (Fedde) G. B. Ownbey (crested pricklypoppy) - SSBC; A; H; AB6793

\section{Passifloraceae}

Passiflora lutea L. (yellow passionflower) QMJV-M; P; H; AB-6264

\section{Phytolaccaceae}

Phytolacca americana L. (American pokeweed) DAOF; P; H; AB

\section{Plantaginaceae}

Plantago heterophylla Nutt. (slender plantain) SSBC; A; H; AB-6937

P. patagonica Jacq. (wooly plantain) - SSBC; A; $\mathrm{H} ; \mathrm{AB}-6859$

P. rhodosperma Dcne. (redseed plantain) SSBC; A; H; AB-6837

\section{Polygalaceae}

Polygala alba Nutt. (white milkwort) - SSBC; P; $\mathrm{H}$; AB-6224

\section{Polygonaceae}

Eriogonum annuum Nutt. (annual buckwheat) SSBC; A; H; AB-6256

E. longifolium Nutt. (longleaf buckwheat) SSBC; P; H; AB-6262

Polygonum aviculare L. (prostrate knotweed) DAOF; A; H; AB-6609

P. lapathifolium L. (curlytop knotweed) WETL; A; H; AB-6895 
P. punctatum Ell. (dotted smartweed) - WETL; A; $\mathrm{H}$; AB-6563

P. scandens $\mathrm{L}$. (climbing false buckwheat) -

QMJV-M; P; H; AB-6605

P. virginianum L. (jumpseed) - QMJV-M; P; H;

AB-6214

Rumex crispus L.* (curly dock) - DAOF,

WETL; P; H; AB-6780

\section{Portulacaceae}

Portulaca pilosa L. (kiss me quick) - DAOF; A; $\mathrm{H}$; AB-6304

\section{Ranunculaceae}

Clematis pitcheri Torr. \& Gray (bluebill) -

QMJV-M; P; H; AB-6819

Delphinium carolinianum Walt. subsp. virescens

(Nutt.) Brooks (Carolina larkspur) - SSBC;

P; $\mathrm{H}$; AB-6802

\section{Rosaceae}

Geum canadense Jacq. (white avens) - QMJVM; P; H; AB-6809

Prunus angustifolia Marsh. (Chickasaw plum) -

DAOF, SSBC; P; S; AB-6277

P. mexicana S. Wats. (Mexican plum) - QMJV$\mathrm{X} ; \mathrm{P} ; \mathrm{T} ; \mathrm{AB}-6557$

\section{Rubiaceae}

Cephalanthus occidentalis L. (buttonbush) -

WETL; P; S; AB-6228

Galium aparine L. (stickywilly) - QMJV-M; A; $\mathrm{H}$; AB-6765

G. circaezans Michx. (licorice bedstraw) -

QMJV-M; P; H; AB-6810

G. virgatum Nutt. (southwestern bedstraw) -

DAOF; A; H; AB-6856

Hedyotis nigricans (Lam.) Fosberg

(diamondflowers) - SSBC; P; H; AB-6240

Houstonia pusilla Schoepf (tiny bluet) - DAOF;

A; H; AB-6912

Sherardia arvensis L.* (blue fieldmadder) -

DAOF; A; H; AB-6843

\section{Rutaceae}

Ptelea trifoliata L. (common hoptree) - SSBC; P; T; AB-6770

Zanthoxylum birsutum Buckl. (Texas Hercules' club) - QMJV-M; P; T; AB-6820

\section{Salicaceae}

Populus deltoides Bartr. ex Marsh. (eastern cottonwood) - WETL; P; T; AB-6200

Salix exigua Nutt. (sandbar willow) - WETL; P; T; AB-6561

S. nigra Marsh. (black willow) - WETL; P; T;

AB-6267

\section{Santalaceae}

Comandra umbellata (L.) Nutt. subsp. pallida (A. DC.) Piehl (pale bastard toadflax) - SSBC; P; H; AB-6866

Sapindaceae

Sapindus drummondii Hook. \& Arn. (soapberry) - QMJV-M; P; T; AB-6280

\section{Sapotaceae}

Sideroxylon lanuginosum Michx. (gum bully) -

QMJV-X; P; T; AB-6890

\section{Scrophulariaceae}

Agalinis densiflora (Benth.) Blake (Osage false foxglove) - SSBC; A; H; AB-6570

A. beterophylla (Nutt.) Small ex Britt. (prairie false foxglove) - SSBC; A; H; AB-6569

Castilleja indivisa Engelm. (Indian paintbrush) SSBC; A; H; AB-6259

Nuttallanthus texanus (Scheele) D. A. Sutton (Texas toadflax) - DAOF, SSBC; A; H; AB-684

Penstemon cobaea Nutt. (cobaea beardtongue) SSBC; P; H; AB-6783

Veronica arvensis L.* (corn speedwell) - DAOF; A; H; AB-6941

$V$. peregrina L. (neckweed) - DAOF; A; H; AB6857 


\section{Solanaceae}

Physalis cinerascens (Dunal) A. S. Hitchs.

(smallflower groundcherry) - DAOF; P;

$\mathrm{H}$; AB-6814

P. heterophylla Nees (clammy groundcherry) -

DAOF; P; H; AB-6835

$P$. longifolia Nutt. (longleaf groundcherry) -

DAOF; P; H; AB-6589

Solanum dimidiatum Raf. (western horsenettle) -

DAOF; A; H; AB-6831

S. elaeagnifolium Cav. (silverleaf nightshade) -

DAOF; P; H; AB-6197

\section{Ulmaceae}

Celtis laevigata Willd. var. reticulata (Torr.) L. Benson (netleaf hackberry) - QMJV-X, SSBC; P; T; AB-6591

C. laevigata Willd. var. texana Sarg. (Texan sugarberry) - QMJV-M; P; T; AB-6838

Ulmus americana L. (American elm) - QMJVM; P; T; AB-6266

U. parvifolia Jacq.* (Chinese elm) - DAOF; P; T; AB-7153

U. pumila L.* (Siberian elm) - DAOF; P; T; AB-6789

U. rubra Muhl. (slippery elm) - DAOF; P; T; AB-6208

\section{Valerianaceae}

Valerianella radiata (L.) Dufr. (beaked cornsalad) - WETL; A; H; AB-6863

\section{Verbenaceae}

Phryma leptostachya L. (American lopseed) QMJV-M; P; H; AB-6898

Phyla nodiflora (L.) Greene (turkey tangle fogfruit) - QMJV-M; P; H; AB-6812

Verbena bracteata Lag. \& Rodr. (bigbract verbena) - DAOF, SSBC; A; H; AB-6865

V. urtricifolia $\mathrm{L}$. (white vervain) - WETL; A; H; AB-6217

\section{Violaceae}

Viola bicolor Pursh (johnny jump-up) - DAOF; A; H; AB-6915

\section{Viscaceae}

Phoradendron tomentosum (DC.) Engelm. ex Gray (Christmas mistletoe) - QMJV-M, QMJV-X; P; S; AB-6839

\section{Vitaceae}

Ampelopsis cordata Michx. (heartleaf peppervine) - WETL; P; V; AB-6196

Parthenocissus quinquefolia (L.) Planch. (Virginia creeper) - QMJV-M; P; V; AB-6276

Vitis acerifolia Raf. (mapleleaf grape) - WETL; P; V; AB-6582

$V$. aestivalis Michx. (summer grape) - QMJVM; P; V; AB-6817a

$V$. cinerea (Engelm.) Millard var. cinerea (graybark grape) - QMJV-M; P; V; AB6930

V. vulpina L. (frost grape) - QMJV-M; P; V; AB-6818

\section{Liliopsida}

\section{Agavaceae}

Yucca glauca Nutt. (soapweed) - SSBC; P; T; AB-6796

\section{Araceae}

Arisaema dracontium (L.) Schott (green dragon) - QMJV-M; P; H; AB-6782

\section{Commelinaceae}

Commelina erecta L. var. angustifolia (Michx.) Fern. (whitemouth dayflower) - QMJVM; P; H; AB-6575

Tradescantia occidentalis (Britt.) Smyth (prairie spiderwort) - SSBC; P; H; AB-6829

\section{Cyperaceae}

Carex albicans Willd. ex Spreng. (whitetinge sedge) - QMJV-X; P; G; AB-6952

C. blanda Dewey (eastern woodland sedge) QMJV-M; P; G; AB-6950

C. brevior (Dewey) Mackenzie (shortbeak sedge) - QMJV-M; P; G; AB-6951

C. leavenworthii Dewey (Leavenworth's sedge) QMJV-M; P; G; AB-6870 
C. oligocarpa Schkuhr ex Willd. (richwoods sedge) - QSJV-M; P; G; AB-6869

Cyperus acuminatus Torr. \& Hook. ex Torr. (tapertip flatsedge) - DAOF, SSBC; P; G; AB-6868

C. lupulinus (Spreng.) Marcks (Great Plains flagsedge) - DAOF, SSBC; P; G; AB-7165

C. schweinitzii Torr. (Schweinitz's flatsedge) SSBC; P; G; AB-6579

Fimbristylis puberula (Michx.) Vahl (hairy fimbry) - SSBC; P; G; AB-6949

\section{Iridaceae}

Sisyrinchium angustifolium P. Mill. (narrowleaf blue-eyed grass) - DAOF; P; H; AB-6864

\section{Juncaceae}

Juncus bufonius L. (toad rush) - WETL; A; G; AB-6867

J. tenuis Willd. (poverty rush) - QMJV-M; P; G; AB-7157

\section{Liliaceae}

Allium canadense L. var. fraseri Ownbey (fraser meadow garlic) - SSBC; P; H; AB-6807

Polygonatum biflorum (Walt.) Ell. (smooth Solomon's seal) - QMJV-M; P; H; AB6794

\section{Orchidaceae}

Spiranthes lacera (Raf.) Raf. (northern slender ladies'-tresses) - SSBC; P; H; AB-6581

\section{Poaceae}

Agrostis byemalis (Walt.) B. S. P. (winter bentgrass) - WETL; P; G; AB-6936

Andropogon gerardii Vitman (big bluestem) SSBC; P; G; AB-6892

Aristida longespica Poir. var. geniculata (Raf.) Fern. (slimspike threeawn) - DAOF; A; G; AB-6594

A. oligantha Michx. (prairie threeawn) DAOF, SSBC; A; G; AB-6622

Bothriochloa laguroides (DC.) Herter (silver beardgrass) - DAOF; P; G; AB-7163

Bouteloua curtipendula (Michx.) Torr. (sideoats grama) - SSBC; P; G; AB-6884
B. hirsuta Lag. (hairy grama) - SSBC; P; G; AB6245

Bromus catharticus Vahl* (rescuegrass) - DAOF; A; G; AB-6910

B. japonicus Thumb. ex Murr.* (Japanese brome) - DAOF; A; G; AB-6826

B. pubescens Muhl. ex Willd. (hairy woodland brome) - QMJV-M; P; G; AB-6778

B. tectorum L.* (cheatgrass) - DAOF; A; G; AB-6920

Cenchrus spinifex Cav. (coastal sandbur) DAOF; A; G; AB-6612

Chasmanthium latifolium (Michx.) Yates (Indian woodoats) - QMJV-M; P; G; AB-6235

Chloris verticillata Nutt. (tumble windmill grass) - DAOF; P; G; AB-6231

Cynodon dactylon (L.) Pers.* (bermudagrass) DAOF; P; G; AB-6891

Dactylis glomerata L.* (orchardgrass) - DAOF; P; G; AB-6798

Dichanthelium aciculare (Desv. ex Poir.) Gould \& C. A. Clark (needleleaf rosette grass) QMJV-M; P; G; AB-6586

D. malacophyllum (Nash) Gould (softleaf rosette grass) - QMJV-M; P; G; AB-6836

D. oligosanthes (J. A. Schultes) Gould var. scribnerianum (Nash) Gould (Scribner's rosette grass) - SSBC; P; G; AB-6946

Digitaria cognata (J. A. Schultes) Pilger (Carolina crabgrass) - DAOF; WETL; P; G; AB-6626

Echinochloa muricata (Beauv.) Fern.* (rough barnyardgrass) - WETL; A; G; AB-6587

Elymus canadensis L. (Canada wildrye) - QMJVM; P; G; AB-7162

Eragrostis curtipedicellata Buckl. (gummy lovegrass) - SSBC; P; G; AB-6879

E. intermedia A. S. Hitchc. (plains lovegrass) SSBC; P; G; AB-6944

E. secundiflora J. Presl. subsp. oxylepis (Torr.) S. D. Koch (red lovegrass) - DAOF; P; G; AB-6613

E. sessilispica Buck. (tumble lovegrass) - SSBC; P; G; AB-6878 
E. spectabilis (Pursh) Steud. (purple lovegrass) SSBC; P; G; AB-6904

E. trichodes (Nutt.) Wood (sand lovegrass) SSBC; P; G; AB-6602

Hordeum pusillum Nutt. (little barley) - DAOF; A; G; AB-6779

Leersia virginica Willd. (whitegrass) - QMJV-M; P; G; AB-6628

Lolium perenne L.* (perennial ryegrass) -

DAOF; P; G; AB-6824

Mublenbergia bushii Pohl (nodding muhly) QMJV-M; P; G; AB-6624

M. sylvatica Torr. ex Gray (woodland muhly) QMJV-M; P; G; AB-6603

Panicum anceps Michx. (beaked panicgrass) QMJV-M; P; G; AB-6227

P. capillare L. (witchgrass) - DAOF; A; G; AB6903

P. virgatum L. (switchgrass) - DAOF, WETL; P; G; AB-6270

Paspalum dilatatum Poir.* (Dallisgrass) - SSBC; P; G; AB-7150

P. setaceum Michx. (thin paspalum) - WETL; P; $\mathrm{G} ; \mathrm{AB}-6621$

Phalaris caroliniana Walt. (Carolina canarygrass) - WETL; A; G; AB-6925

Phragmites australis (Cav.) Trin. Ex Steud.* (common reed) - WETL; P; G; AB-7160

Poa annua L.* (annual bluegrass) - DAOF; A; $\mathrm{G} ; \mathrm{AB}-6928$

P. arachnifera Torr. (Texas bluegrass) - SSBC; P; G; AB-6909

P. chapmaniana Scribn. (Chapman's bluegrass) SSBC; A; G; AB-6808

Saccharum giganteum (Walt.) Pers. (sugarcane plumegrass) - WETL; P; G; AB-6953

Schedonnardus paniculatus (Nutt.) Trel. (tumblegrass) - DAOF, SSBC; P; G; AB6882
Schedonorus phoenix (Scop.) Holub.* (tall fescue) - DAOF; P; G; AB-6813

Schizachyrium scoparium (Michx.) Nash (little bluestem) - QMJV-X, SSBC; P; G; AB6251

Setaria pumila (Poir.) Roemer \& J.A. Schultes (yellow foxtail) - DAOF; A; G; AB-6583)

S. viridis (L.) Beauv.* (green bristlegrass) DAOF; A; G; AB-6873

Sorghastrum nutans (L.) Nash (Indiangrass) SSBC; P; G; AB-6248

Sorghum halepense (L.) Pers.* (Johnsongrass) DAOF; P; G; AB-7151

Spartina pectinata Bosc ex Link (prairie cordgrass) - WETL; P; G; AB-6203

Sporobolus cryptandrus (Torr.) Gray (sand dropseed) - DAOF, SSBC; P; G; AB-6627

S. vaginiflorus (Torr. ex Gray) Wood var. ozarkanus (Fern.) Shinners (Ozark dropseed) - SSBC; P; G; AB-6625

Tridens flavus (L.) A. S. Hitchc. (purpletop tridens) - DAOF, QMJV-X; P; G; AB6242

Vulpia elliotea (Raf.) Fern. (squirreltail fescue) DAOF; A; G; AB-6935a

\section{Smilacaceae}

Smilax bona-nox L. (saw greenbriar) - QMJVM, QMJV-X; P; H; AB-6211

S. rotundifolia L. (roundleaf greenbriar) -

QMJV-M, QMJV-X; P; H; AB-7154

S. tamnoides L. (bristly greenbriar) - QMJV-M; P; H; AB-6556

\section{Typhaceae}

Typha domingensis Pers. (Southern cattail) WETL; P; H; AB-6875 